obstruction, since the bile is the main route for the excretion of copper, this is rendered unlikely by the findings of Hunt and his colleagues that in cases of longstanding extra-hepatic biliary obstruction the amount of copper in the liver was only about twice the normal. But in this type of case reabsorption of copper by the bile duct's mucosa and excretion via the kidney might explain the lower level of hepatic copper. Measuring the copper content of the liver in cases of long-standing viral hepatitis (in which the anatomical level of biliary obstruction is presumably about the same as in idiopathic biliary cirrhosis) should help to answer this question.

In a group of 18 cases of non-biliary cirrhosis studied by Hunt and his colleagues the mean content of iron $(282 \mu \mathrm{g} . / \mathrm{g}$.) was not significantly greater than normal $(183 \mu \mathrm{g} / \mathrm{g}$.$) . However, other workers have obtained$ different figures ${ }^{4}$-figures which led Drs. Sheila Callender and J. S. Malpas to study the absorption of iron in patients with cirrhosis. Their findings are reported in the B.M.J. this week at page 1516. In nine cirrbotic patients, despite an abnormally high level of serum iron, the absorption of iron from the bowel was significantly increased whether administered as inorganic ferrous sulphate or as rabbit haemoglobin. In the absence of adequate information about the mechanism normally controlling the absorption of iron it is impossible to explain where the defect lies in these cirrhotics. The effect of giving pancreatic extracts with the test doses of iron did, however, produce some highly suggestive results. In each instance the absorption of iron was diminished. Pancreatic deficiency in patients with cirrhosis of the liver has been described by other observers $^{6}$ " and may therefore, as suggested by Callender and Malpas, be causing the excessive uptake of iron they detected.

The possibility that patients with cirrhosis of the liver have an abnormally high uptake of heavy metals, as shown by Callender and Malpas for iron, and as suggested by the figures of Hunt and his colleagues for copper, raises another question of some importance. Does the increase of heavy metals in the liver have anything to do with the fact that primary carcinoma is commoner in cirrhotic than non-cirrhotic livers? This increase of malignant hepatomas has been reported from many centres and is confirmed by the latest figures from St. Thomas's Hospital reported by Drs. S. G. Elkington, D. J. McBrien, and H. Spencer at page 1501 of the B.M.J. In a series of 7,366 necropsies performed in the 23 years from 1940 to 1962 inclusive there were 39 cases of primary hepatic carcinoma and 157 cases of cirrhosis. Thirty of the carcinomata developed in association with cirrhosis, an incidence of $19 \%$, the remaining 9 occurring amongst the 7,209 non-cirrhotic cases, an incidence of $0.125 \%$. The incidence in the cirrhotic livers was therefore 152 times that in the noncirrhotic livers. Data at present available are insufficient to justify any firm conclusions, but Hunt and his colleagues are undoubtedly correct in concluding that "the role of minute quantities of heavy metals in the aetiology of certain types of cirrhosis may be appreciable. Their part in the causation of cancer of the liver remains to be investigated."

\section{MILROY'S DISEASE AND LYMPHOEDEMA}

Oedema caused by obstruction of the lymphatics may be secondary to neoplasm, trauma, or infection. In primary lymphoedema the cause of lymphatic obstruction is not evident and cases may be classified arbitrarily as congenital (present at birth), praecox (onset before $35)$, and late.

W. F. Milroy ${ }^{1}$ described a family in which 22 of 97 members in six generations had chronic oedema of one or both legs. The swelling had been present from birth in all cases except one. The condition was compatible with longevity and good health. Further examples of hereditary oedema were described by E. E. Letessier, ${ }^{2}$ M. Nonne, ${ }^{3}$ and $\mathrm{H}$. Meige. ${ }^{4}$ Hence the complex eponym Nonne-Milroy-Meige's disease. However, most cases of chronic lymphoedema of the legs are neither familial nor congenital. In one series of 300 cases of chronic lymphoedemas 105 were primary : twelve of these were congenital but none was familial; the remaining 93 were praecox. In another 107 cases, reported by J. B. Kinmonth and colleagues, ${ }^{6} 12$ were congenital, 82 were praecox, and 13 of late onset. In this series 18 were familial and only 2 both familial and congenital. Thus only about $1 \%$ of cases of primary lymphoedema are true examples of Milroy's disease-that is, are both hereditary and congenital.

\footnotetext{
Milroy, W. F., N.Y. med. J., 1892. 56, 505

(a) Strasbourg, 1865, quoted by Schroeder and Helweg-Larsen.21

3 Nonne, M., Virchows Arch. path. Anat., 1891, 125, 189.

4 Meige, H., Presse mid., 1898, 6. 341.

Allen, E. V. Barker, N. W.. and Hines, E. A., Peripheral Vascular Diseases, 3rd ed.. 1962, p. 690. Saunders, Philadelphia and I ondon.

Kinmonth. J. B., Taylor. G. W., Tracy, G. D., and Marsh, J. D., Brit. J. Surg. $1957,45,1$.

7 Hope, W. B., and French, H., Quart. J. Med., 1908, 1, 312.

- Hagy, G. W... and Danhof. I., Amer. J. hum. Genet., 1958, 10, 141.

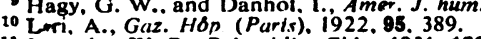

11 Jaroschy, W. B., Britr. klin. Chir. 1931, i52, 632

12 Weber. F. P., and Schititer, A.. Proc. roy. Soc. Med., 1937, 30, 933.

13 Bloom. D., N.Y. Si. J. Med. i941. 41. 856.

14 Polani, P. E.. Brit. med. Bulli, 1961, 17, 200.

18 Jones, H. E., Arih. Dis. Childh. 1960, 35, 192.

16 Gough. M. H. Guiney, E. J. and Kinmo

10 Jennett. J. H.. Clin. Orihop., 1956. 8. 122.

19 McGuire. J.. and Zeek. P.. J. Amer. med. Ass. 1932, 88, 870.

20 Radner, S., Acra derm.-venereol. (Srockh.), 1946, 26, 261.

Schroeder, E., and Helweg-l.arsen. H.. d.to med scand. 1950, 137,198

${ }^{2}$ Cockayne. E. A.. Inhorited Ahnormalities of the Skin and its Appenduges, 1933 p. 375. Oxford Univ. Prens. London.

Barker. N. W.. Carey, B.. and Brough, W., Minn. Med.. 1959, 42, 227.

24 Dencker, S. J., and Gott ries. I.. Aclu med. scand. 1954, 150, 277.

23 Panos. T. C.. J. Amer. med. Ass.. 1956, 161. 1475.

22 Kowlem, R., B-it. J. plast. Surg.. 1948. 1, 48. Simeone, F. A., 1962, p. 363 . London.
} 
In primary lymphoedema swelling often starts at one ankle and within months or years spreads up the limb before appearing in the other limb in a half to two-thirds of the cases. ${ }^{5}$ In Milroy's patients the swelling did not extend above the inguinal ligament, but in about $10 \%$ of cases other parts of the body are affected, especially the upper limbs and face. Patients often attribute the onset of swelling to some precipitating factor, usually traumatic, but this is generally trivial and probably coincidental in most cases. While initially the oedema is soft and pitting and the overlying skin normal, later the subcutaneous tissues may become fibrotic, the skin thick and hyperkeratotic, and the oedema non-pitting. Usually swelling is minimal on waking and maximal at night. Recurrent attacks of "lymphangitis" occur in about one-quarter of the patients $^{5-9}$ and have been attributed to streptococcal or staphylococcal infection.

Among Kinmonth and his colleagues' 107 patients with primary chronic lymphoedema ${ }^{6} 14(13 \%)$ had associated non-lymphatic congenital abnormalities, of which eight involved blood-vessels or the heart. Several other associations of this kind have been reported. ${ }^{70-13}$ A well-defined association with non-hereditary congenital lymphoedema is ovarian dysgenesis, and in these cases the women often have other anomalies such as webbing of the neck, coarctation of the aorta, and dwarfism. ${ }^{14}$ Their cells often lack the Barr body (i.e., are chromatin-negative) and have abnormal sex chromosomes, the most common defect being the presence of only one instead of the normal two $\mathrm{X}$ chromosomes. Swelling may involve the hands, and usually disappears in a few months or years, ${ }^{15}$ but sometimes it recurs after the administration of oestrogens and persists until late childhood and adolescence.

Lymphangiography has been informative in diagnosis. Kinmonth and his associates ${ }^{6}{ }^{16}$ have used direct inspection after subcutaneous injection of a blue dye and radiography after the injection of radio-opaque substances into lymph trunks. In cases of primary lymphoedema they have demonstrated hypoplasia of the lymphatic trunks in $55 \%$, varicose dilatation in $24 \%$, aplasia in $14 \%$, and backflow of dye into the lymphatic dermal plexus (indicating lymphatic obstruction) in $6 \%$. On the basis of these findings they suggested that primary lymphoedema is due to congenital underdevelopment of the lymphatics. Other theories include increased capillary permeability ${ }^{17-20}$ and arteriolar disease. ${ }^{21}$ Infection in these cases is thought to be secondary to the lymphoedema and not causative. About three-quarters of the patients with primary lymphoedema whose cases have been reported are females. ${ }^{56}$ In hereditary cases transmission is usually of the autosomal dominant type, but sex-linkage has been suggested for some cases. ${ }^{921} 22$ Most patients are not excessively troubled by their condition. In early cases elevation of the limbs in combination with diuretics such as chlorothiazide ${ }^{23}$ may help to control oedema, as may rubber bandages rolled over cotton stockings. Reports on the value of treatment with steroids have been conflicting. ${ }^{182425}$ When oedema is massive, surgical removal of the swollen subcutaneous tissues may be indicated and plastic operations are usually undertaken, but operations which aim at making new pathways for carrying lymph have been disappointing. ${ }^{26} 27$

\section{HARMLESS FOOD}

In the last decade British legislation to maintain pure food has undergone a revolution. Once a list of prohibited additives left virtually everything else free to be added unless its use was obviously injurious to the health of the consumer. Now only substances specifically permitted for use may be added to food. Food technologists are all the time developing new methods of treating food, so that the Food Standards Committee, with its Subcommittee on Food Additives and Contaminants, keeps each group of substances under continual review, and it hopes to issue, after hearing representations from potential users, revised rulings on each group of additives at intervals of about five years.

Its latest report $^{1}$ considers antioxidants which are added to fat to prevent rancidity and so to prolong the storage period both of bulk fats and of foods containing fat. Representations from users of new materials have been carefully scrutinized. Of five substances proposed for use the introduction of four was rejected on the grounds that no satisfactory case had been made for the need to use them. Of the previously permitted antioxidants butylated hydroxy toluene (B.H.T.) had come under suspicion because of reactions seen in rats receiving high-fat diets containing this substance. By comparison these rats reacted less favourably than rats on a similar diet containing the closely related butylated hydroxyanisole (B.H.A.). There was additional evidence that rats metabolized B.H.A. more rapidly and completely than they did B.H.T. Recent work has shown that in the metabolism of B.H.A. man more closely resembles the rat than the dog. ${ }^{2}$ The committee therefore recommends that B.H.T. be removed from the list of antioxidants to be permitted in food. The report amply illustrates the care with which anything that might remotely attract suspicion because of an adverse reaction in tests on laboratory animals may be removed from the list of permitted additives.

The main problem in this work is the design of proper tests on animals that will provide information which can be useful in evaluating any possible hazards that a substance might present to the human consumer. It is

1 Food Standards Committee Report on Antioxidants in Food, 1963. H.M.S.O 2 Astill, B. D., et al., J. Agric. Food Chem., 1962, 10, 315. 